\title{
KERNEL DENSITY ESTIMATION FOR LINEAR PROCESSES WITH $\psi$-WEAKLY DEPENDENT INNOVATIONS: ASYMPTOTIC NORMALITY
}

\section{KAOUTHAR EL FASSI and LAHCEN DOUGE}

FSTT

University Abdelmalik Essaadi

B. P. 416 Tangier

Morocco

e-mail: k.elfassi@gmail.com

FSTG

University Cadi Ayyad

B. P. 549 Marrakech

Morocco

e-mail: lahcen.douge@gmail.com

\begin{abstract}
In this paper, we study the kernel estimate of the density function of linear processes with $\psi$-weakly dependent innovations. The asymptotic normality is shown under general conditions and some conditions on the decay of the weak dependence coefficients. Some numerical results based on simulations are also presented and discussed.
\end{abstract}

2010 Mathematics Subject Classification: 62G07, 62G20.

Keywords and phrases: linear process, $x$-weakly dependence, kernel estimate.

Received July 29, 2019; Revised September 30, 2019

(C) 2019 Scientific Advances Publishers 


\section{Introduction}

Let $X_{1}, X_{2}, \ldots$, be an identically distributed sequence of a linear process $X_{i}=\sum_{r=0}^{\infty} a_{r} Z_{i-r}, i \geq 1$, where $\left\{Z_{t}\right\}_{t \in \mathbb{Z}}$ is a strictly stationary sequence of $x$-weakly dependent random variables with mean zero and finite variance, and $\left\{a_{r}\right\}_{r \in \mathbb{N}}$ is a sequence of real numbers.

Doukhan and Louhichi [4] have introduced the concept of $x$-weakly dependence which generalizes the notions of mixing and association. A sequence of random variables $\left(Z_{i}\right)_{i \in \mathbb{Z}}$ with values in $\mathbb{R}$ is called $\psi$-weakly dependent if there exists a sequence $\left(\theta_{r}\right)_{r \in \mathbb{N}}$ decreasing to zero at infinity such that, for any $k$-tuple $\left(s_{1}, \ldots, s_{k}\right)$ and any $l$-tuple $\left(t_{1}, \ldots, t_{l}\right)$ with $s_{1} \leq \ldots \leq s_{k}<s_{k}+r=t_{1} \leq \ldots \leq t_{l}$ and for any bounded Lipschitz functions $g: \mathbb{R}^{k} \rightarrow \mathbb{R}$ and $h: \mathbb{R}^{l} \rightarrow \mathbb{R}$ with $\|g\|_{\infty} \leq 1$ and $\|h\|_{\infty} \leq 1$, one has

$$
\left|\operatorname{Cov}\left(g\left(Z_{s_{1}}, \ldots, Z_{s_{k}}\right), h\left(Z_{t_{1}}, \ldots, Z_{t_{l}}\right)\right)\right| \leq \psi(k, l, \operatorname{Lip}(g), \operatorname{Lip}(h)) \theta_{r} .
$$

Here Lip $(g)$ denotes the Lipschitz modulus of $g$, that is,

$$
\operatorname{Lip}(g)=\sup _{x \neq y} \frac{|g(x)-g(y)|}{\|x-y\|_{1}}, \quad\|x\|_{1}=\sum_{s=1}^{k}\left|x_{s}\right| \quad \text { for } x=\left(x_{1}, \ldots, x_{k}\right) \in \mathbb{R}^{k},
$$

and $\psi: \mathbb{N}^{2} \times \mathbb{R}_{+}^{2} \rightarrow \mathbb{R}_{+}$is an appropriate function.

In order to consider a large families of dependent random variables including associated, mixing, Gaussian and Markov processes (we refer to Doukhan and Louhichi [4] for more examples), we will suppose that

$$
\psi(u, v, \operatorname{Lip}(f), \operatorname{Lip}(g)) \leq c u v \max (\operatorname{Lip}(f) \operatorname{Lip}(g), \operatorname{Lip}(f), \operatorname{Lip}(g), 1),
$$

where $c$ is a positive constant. Notice that if $\left(Z_{i}\right)_{i \in \mathbb{Z}}$ is a sequence of associated or Gaussian random variables, then $\left(Z_{i}\right)_{i \in \mathbb{Z}}$ is $\psi$-weakly dependent with 
$\theta_{r}=\sup _{i} \sum_{j:|i-j|>r}\left|\operatorname{Cov}\left(Z_{i}, Z_{j}\right)\right|$ and $\psi(u, v, \operatorname{Lip}(f), \operatorname{Lip}(g))=\min (u, v)$

$\times \operatorname{Lip}(f) \operatorname{Lip}(g)$.

If the sequence $\left(Z_{i}\right)_{i \in \mathbb{Z}}$ is a strongly mixing, then it is $\psi$-weakly dependent with

$$
\theta_{r}=\alpha_{r} \quad \text { and } \quad \psi(u, v, \operatorname{Lip}(f), \operatorname{Lip}(g))=4\|f\|_{\infty}\|g\|_{\infty} .
$$

The strong mixing coefficients, $\left(\alpha_{r}\right)_{r \in \mathbb{N}}$, of $\left(Z_{i}\right)_{i \in \mathbb{Z}}$ are defined by

$$
\alpha_{r}=\sup _{k \in \mathbb{Z}} \sup _{(A, B) \in \mathcal{A}_{k} \times \mathcal{B}_{k+r}}\left|\operatorname{Cov}\left(\mathbb{1}_{A}, 1_{B}\right)\right|,
$$

where

$$
\mathcal{A}_{k}=\sigma\left(Z_{i}, i \leq k\right) \quad \text { and } \quad \mathcal{B}_{k}=\sigma\left(Z_{i}, i \geq k\right) .
$$

$1_{A}$ denotes the indicator function of the event $A$.

Assume that $X_{1}$ has a probability density $f$ and, for any $1 \leq i, j \leq n$, $\left(X_{i}, X_{j}\right)$ has a probability density $f_{i, j}$. Further, let $\left\{h_{n}\right\}_{n \geq 0}$ be a sequence of positive constants such that, as $n \rightarrow \infty, h_{n} \rightarrow 0$ and $n h_{n} \rightarrow \infty$. The classical kernel estimator of $f$ is defined as

$$
f_{n}(x):=\frac{1}{n h_{n}} \sum_{i=1}^{n} K\left(\frac{x-X_{i}}{h_{n}}\right), \quad x \in \mathbb{R},
$$

where the kernel $K$ is a bounded function with compact support such that

$$
\int_{\mathbb{R}} K(u) d u=1 .
$$

In this paper, we present the asymptotic normality of the kernel density estimators. The asymptotic normality depends on both the coefficients of linear processes and the behaviour of $\left(\theta_{r}\right)_{r \in \mathbb{N}}$. For linear processes with independent innovations, Chanda [2] and Hallin and Tran 
[6] have obtained the asymptotic normality of $f_{n}(x)$ under some conditions on $\left\{a_{n}\right\}$ and $\left\{h_{n}\right\}$. The asymptotic normality of the kernel density estimators was studied by $\mathrm{Lu}$ [11] for linear processes with $\alpha$-mixing innovations. Several authors considered the asymptotic properties of kernel density estimators for linear processes with short memory or long memory by using the martingale approach initiated by Ho et al. [8]. We cite, for example, Honda [9], Mielniczuk and Wu [14] and Schick and Wefelmeyer [12]. Mielniczuk and Wu [14] improved Hallin and Tran's results by imposing $\sum_{i=0}^{\infty}\left|a_{i}\right|<\infty$ and only the natural conditions on $\left\{h_{n}\right\}$. For other works on density estimation for linear processes, we can refer to Honda [10], Wu et al. [15] and Hamdad et al. [7]. It should be pointed that our result is obtained under the same general conditions on the density function $f$ and the kernel $K$, and the coefficients $\left\{a_{n}\right\}$ as in Hallin and Tran [6] and Lu [11]. Some additional conditions on the bandwidth and the dependence coefficients are assumed.

\section{Notation, Assumptions and Main Result}

Divide the set $\{1, \ldots, n\}$ into $k$ large $p$-blocks, $I_{j}$, and small $q$-blocks, $J_{j}, j=1, \ldots, k$, as follows:

$$
\begin{aligned}
& I_{j}=\{(j-1)(p+q)+1, \ldots,(j-1)(p+q)+p\}, \\
& J_{j}=\{(j-1)(p+q)+p+1, \ldots, j(p+q)\},
\end{aligned}
$$

where $p=p_{n}, q=q_{n}$ are positive integers tending to $\infty$ as $n \rightarrow \infty$ and $k=k_{n}$ is defined by $k=[n /(p+q)]$, where $[x]$ stands for the integral part of $x$. We suppose that

$$
\frac{q^{k}}{n} \rightarrow 0 \quad \text { and } \quad \frac{p^{k}}{n} \rightarrow 1 \quad \text { as } n \rightarrow \infty
$$


Define

$$
\tilde{X}_{i}:=\sum_{r=0}^{s} a_{r} Z_{i-r}, \quad i \geq 1
$$

where $s=s_{n}<q$ is a positive integer tending to $\infty$ as $n \rightarrow \infty$.

Consider the kernel estimator $g_{n}(x)$ defined by

$$
g_{n}(x):=\frac{1}{n h_{n}} \sum_{i=1}^{n} K\left(\frac{x-\tilde{X}_{i}}{h_{n}}\right), \quad x \in \mathbb{R}
$$

For $x \in \mathbb{R}$, set

$$
Z_{n i}(x):=\frac{1}{\sqrt{n h_{n}}}\left(K\left(\frac{x-\tilde{X}_{i}}{h_{n}}\right)-E K\left(\frac{x-\tilde{X}_{i}}{h_{n}}\right)\right),
$$

and

$$
S_{n}:=\sqrt{n h_{n}}\left(g_{n}(x)-E g_{n}(x)\right)=\sum_{i=1}^{n} Z_{n i}(x)
$$

For $j=1, \ldots, k$, let $\eta_{j}, \xi_{j}$ and $\zeta_{k}$ be defined as follows:

$$
\eta_{j}:=\sum_{i \in I_{j}} Z_{n i}(x), \quad \xi_{j}:=\sum_{i \in J_{j}} Z_{n i}(x), \quad \zeta_{k}:=\sum_{i=k(p+q)+1}^{n} Z_{n i}(x)
$$

so that

$$
S_{n}=\sum_{j=1}^{k} \eta_{j}+\sum_{j=1}^{k} \xi_{j}+\zeta_{k}=: T_{n}+T_{n}^{\prime}+T_{n}^{\prime \prime}
$$

In order to formulate our main result, we now list some assumptions. Denote by $C$ (different) constants whose values are allowed to change. Through this paper we consider that all limits are taken as $n \rightarrow \infty$. 


\section{Assumptions}

(A1) For all $x, y \in \mathbb{R}$,

$$
\sup _{i \neq j}\left|f_{i, j}(x, y)-f(x) f(y)\right|<C .
$$

(A2) The kernel $K$ satisfies a Lipschitz condition of order 1 , that is, for all $x, y \in \mathbb{R}$,

$$
|\mathrm{K}(\mathrm{x})-K(y)| \leq C|x-y|
$$

(A3) The sequence $\left\{a_{r}\right\}_{r \in \mathbb{N}}$ satisfies the condition $\left|a_{r}\right|=\mathcal{O}\left(r^{-a}\right)$, for some $a>4$.

(A4) $\theta_{k}=\mathcal{O}\left(k^{-b}\right)$, for some $b>2+\frac{a-2}{2(a-1)}\left(\frac{a+2}{a-3}+\frac{3}{2}+3(a-1)\right)$.

(A5) $n h_{n}^{(a+2) /(a-3)}(\log n)^{-\delta} \rightarrow \infty$, for some $\delta>0$.

(A6) $(\log n)^{\delta(a-3) / 4} n h_{n}^{[2(b-2) /(a-2)-3](a-1)-3 / 2} \rightarrow 0$.

Notice that if $x$ is a Lebesgue point of $f$ and $(x, x)$ is a Lebesgue point of all functions $f_{1, v}, v \geq 2$, by applying Theorem 3 in Chapter 2 of Devroye and Györfi [3], the condition (A1) can be replaced by the condition $\sup _{v \geq 2}\left|f_{1, v}(x, x)\right|<C$. The condition (A2) is a mild condition on the kernel $K$. The conditions (A5) and (A6) hold if the values of $a$ and $b$ are big enough.

Theorem 1. Assume that assumptions (A1)-(A6) hold. Then, for all Lebesgue points $x$ of $f$ such that $f(x)>0$,

$$
\sqrt{n h_{n}}\left(f_{n}(x)-E f_{n}(x)\right) \stackrel{d}{\rightarrow} \mathcal{N}\left(0, \sigma^{2}(x)\right),
$$

where

$$
\sigma^{2}(x)=f(x) \int_{\mathbb{R}} K^{2}(u) d u,
$$

“ $\stackrel{d}{\rightarrow}$ " denotes convergence in distribution. 
We introduce the following lemmas which we need in the proof of the theorem. The first lemma shows that, for all $x \in \mathbb{R}, f_{n}(x)$ and $g_{n}(x)$ have the same asymptotic distribution.

Lemma 1. Assume that assumptions (A2) and (A3) hold. Then, for any $\varepsilon>0$ and for all $x \in \mathbb{R}$,

$$
P\left[\sqrt{n h_{n}}\left|f_{n}(x)-g_{n}(x)\right|>\varepsilon\right] \rightarrow 0 .
$$

Proof. Using (A2), we get

$$
\begin{aligned}
\sqrt{n h_{n}}\left|f_{n}(x)-g_{n}(x)\right| & \leq\left(n h_{n}\right)^{-1 / 2} \sum_{i=1}^{n}\left|K\left(\frac{x-X_{i}}{h_{n}}\right)-K\left(\frac{x-\tilde{X}_{i}}{h_{n}}\right)\right| \\
& \leq C n^{-1 / 2} h_{n}^{-3 / 2} \sum_{i=1}^{n}\left|X_{i}-\tilde{X}_{i}\right| .
\end{aligned}
$$

Then

$$
\begin{aligned}
P\left[\sqrt{n h_{n}}\left|f_{n}(x)-g_{n}(x)\right|>\varepsilon\right] & \leq P\left[\sum_{i=1}^{n}\left|X_{i}-\tilde{X}_{i}\right|>\frac{n^{1 / 2} h_{n}^{3 / 2} \varepsilon}{C}\right] \\
& \leq \sum_{i=1}^{n} P\left[\left|X_{i}-\tilde{X}_{i}\right|>\frac{h_{n}^{3 / 2} \varepsilon}{C n^{1 / 2}}\right] \\
& \leq C n h_{n}^{-3} \varepsilon^{-2} \sum_{i=1}^{n} E\left|X_{i}-\tilde{X}_{i}\right|^{2} \\
& \leq C n h_{n}^{-3} \varepsilon^{-2} \sum_{i=1}^{n} E\left(\sum_{r=s+1}^{\infty} a_{r} Z_{i-r}\right)^{2} \\
& \leq C n^{2} h_{n}^{-3} \varepsilon^{-2} E Z_{1}^{2}\left(\sum_{r=s+1}^{\infty}\left|a_{r}\right|\right)^{2}
\end{aligned}
$$


Choose $s=\left[(\log n)^{\delta(a-3) / 2} n^{2} h_{n}^{-3}\right]^{1 / 2(a-1)}$. Thus, by (A3),

$$
P\left[\sqrt{n h_{n}}\left|f_{n}(x)-g_{n}(x)\right|>\varepsilon\right] \leq C n^{2} h_{n}^{-3} \varepsilon^{-2} s^{-2(a-1)}=\mathcal{O}\left((\log n)^{-\delta(a-3) / 2}\right) .
$$

Lemma 2. Assume that assumptions (A1)-(A2) hold. Then, for all $v \geq 2$ and for all $x \in \mathbb{R}$,

$$
\left|\operatorname{Cov}\left(K\left(\frac{x-\tilde{X}_{1}}{h_{n}}\right), K\left(\frac{x-\tilde{X}_{v}}{h_{n}}\right)\right)\right| \leq C\left[h_{n}^{2}+\frac{1}{h_{n}} \sum_{r=s+1}^{\infty}\left|a_{r}\right|\right] .
$$

Proof. For all $v \geq 2$, by (A1),

$$
\begin{aligned}
& \frac{1}{h_{n}^{2}}\left|\operatorname{Cov}\left(K\left(\frac{x-X_{1}}{h_{n}}\right), K\left(\frac{x-X_{v}}{h_{n}}\right)\right)\right| \\
& \quad=\left|\int_{\mathbb{R}^{2}} K(u) K(w)\left[f_{1, v}\left(x-h_{n} u, x-h_{n} w\right)-f\left(x-h_{n} u\right) f\left(x-h_{n} w\right) d u d w\right]\right| \\
& \quad \leq C .
\end{aligned}
$$

By (A2), for all $v \geq 2$,

$$
\begin{aligned}
& \left|\operatorname{Cov}\left(K\left(\frac{x-X_{1}}{h_{n}}\right), K\left(\frac{x-X_{v}}{h_{n}}\right)\right)-\operatorname{Cov}\left(K\left(\frac{x-\tilde{X}_{1}}{h_{n}}\right), K\left(\frac{x-\tilde{X}_{v}}{h_{n}}\right)\right)\right| \\
& \leq 2|| K \|_{\infty}\left[E\left|K\left(\frac{x-X_{1}}{h_{n}}\right)-K\left(\frac{x-\tilde{X}_{1}}{h_{n}}\right)\right|+E\left|K\left(\frac{x-X_{v}}{h_{n}}\right)-K\left(\frac{x-\tilde{X}_{v}}{h_{n}}\right)\right|\right] \\
& \leq \frac{C}{h_{n}}\left[E\left|X_{1}-\tilde{X}_{1}\right|+E\left|X_{v}-\tilde{X}_{v}\right|\right] \\
& \leq \frac{C}{h_{n}} \sum_{r=s+1}^{\infty}\left|a_{r}\right| .
\end{aligned}
$$


Then

$$
\left|\operatorname{Cov}\left(K\left(\frac{x-\tilde{X}_{1}}{h_{n}}\right), K\left(\frac{x-\tilde{X}_{v}}{h_{n}}\right)\right)\right| \leq C\left[h_{n}^{2}+\frac{1}{h_{n}} \sum_{r=s+1}^{\infty}\left|a_{r}\right|\right] .
$$

Lemma 3. Assume that assumptions (A1)-(A6) hold. Then, for all $x \in \mathbb{R}$,

$$
\frac{1}{h_{n}} \sum_{v=2}^{\infty}\left|\operatorname{Cov}\left(K\left(\frac{x-\tilde{X}_{1}}{h_{n}}\right), K\left(\frac{x-\tilde{X}_{v}}{h_{n}}\right)\right)\right| \rightarrow 0 .
$$

Proof. For $2 \leq v \leq 2 s$, define

$$
X_{v}^{*}:=\sum_{r=0}^{[v / 2]} a_{r} Z_{v-r}
$$

Observe that

$$
\begin{aligned}
\operatorname{Cov}\left(K\left(\frac{x-\tilde{X}_{1}}{h_{n}}\right), K\left(\frac{x-\tilde{X}_{v}}{h_{n}}\right)\right) \\
=E\left[\left(K\left(\frac{x-\tilde{X}_{1}}{h_{n}}\right)-E K\left(\frac{x-\tilde{X}_{1}}{h_{n}}\right)\right)\left(K\left(\frac{x-\tilde{X}_{v}}{h_{n}}\right)-K\left(\frac{x-X_{v}^{*}}{h_{n}}\right)\right)\right] \\
+\operatorname{Cov}\left(K\left(\frac{x-\tilde{X}_{1}}{h_{n}}\right), K\left(\frac{x-X_{v}^{*}}{h_{n}}\right)\right) .
\end{aligned}
$$

Using (A2) and the boundedness of $K$, we obtain

$$
\begin{aligned}
& \left|E\left[\left(K\left(\frac{x-\tilde{X}_{1}}{h_{n}}\right)-E K\left(\frac{x-\tilde{X}_{1}}{h_{n}}\right)\right)\left(K\left(\frac{x-\tilde{X}_{v}}{h_{n}}\right)-K\left(\frac{x-X_{v}^{*}}{h_{n}}\right)\right)\right]\right| \\
& \leq \frac{C}{h_{n}} E\left|\tilde{X}_{v}-X_{v}^{*}\right| \\
& \quad \leq \frac{C}{h_{n}} \sum_{r=[v / 2]+1}^{\infty}\left|a_{r}\right| .
\end{aligned}
$$


For $n$ large enough such that $h_{n} \leq 1$, by the $\psi$ weak dependence of $\left(Z_{t}\right)_{t \in \mathbb{Z}}$,

$$
\left|\operatorname{Cov}\left(K\left(\frac{x-\tilde{X}_{1}}{h_{n}}\right), K\left(\frac{x-X_{v}^{*}}{h_{n}}\right)\right)\right| \leq C \frac{s v}{h_{n}^{2}} \theta_{v-[v / 2]-1} .
$$

Now, for $v \geq 2 s$,

$$
\left|\operatorname{Cov}\left(K\left(\frac{x-\tilde{X}_{1}}{h_{n}}\right), K\left(\frac{x-\tilde{X}_{v}}{h_{n}}\right)\right)\right| \leq C \frac{s^{2}}{h_{n}^{2}} \theta_{v-s-1}
$$

Let $m=m(n)=\left[h_{n}^{\theta-1}\right]$ with $\theta$ is an arbitrary number such that $0<\theta<1$ and $\frac{2}{a-2}<1-\theta<\frac{1}{a-1}\left(\frac{a+2}{a-3}+\frac{3}{2}\right)$. By (A5), we have, for $n$ large enough, $m<s$. Then

$$
\begin{aligned}
& \sum_{v=2}^{\infty}\left|\operatorname{Cov}\left(K\left(\frac{x-\tilde{X}_{1}}{h_{n}}\right), K\left(\frac{x-\tilde{X}_{v}}{h_{n}}\right)\right)\right| \\
& =\sum_{v=2}^{m}\left|\operatorname{Cov}\left(K\left(\frac{x-\tilde{X}_{1}}{h_{n}}\right), K\left(\frac{x-\tilde{X}_{v}}{h_{n}}\right)\right)\right|+\sum_{v=m+1}^{2 s}\left|\operatorname{Cov}\left(K\left(\frac{x-\tilde{X}_{1}}{h_{n}}\right), K\left(\frac{x-\tilde{X}_{v}}{h_{n}}\right)\right)\right| \\
& \quad+\sum_{v=2 s+1}^{\infty}\left|\operatorname{Cov}\left(K\left(\frac{x-\tilde{X}_{1}}{h_{n}}\right), K\left(\frac{x-\tilde{X}_{v}}{h_{n}}\right)\right)\right| .
\end{aligned}
$$

Using (A3) and (A4), by (1), (3), (4), (5), and (6), we get, for $n$ large enough,

$$
\begin{aligned}
& \frac{1}{h_{n}} \sum_{v=2}^{\infty}\left|\operatorname{Cov}\left(K\left(\frac{x-\tilde{X}_{1}}{h_{n}}\right), K\left(\frac{x-\tilde{X}_{v}}{h_{n}}\right)\right)\right| \\
& \left.\leq C\left[h_{n}^{\theta}+h_{n}^{-3+\theta} \sum_{r=s+1}^{\infty}\left|a_{r}\right|\right]+C h_{n}^{-2}\left[\sum_{v=m+1}^{2 s} \sum_{r=[v / 2]+1}^{\infty}\left|a_{r}\right|+s h_{n}^{-1} \sum_{v=m+1}^{2 s} v \theta_{v-[v / 2]-1}\right)\right] \\
& \quad+C h_{n}^{-3} s^{2} \sum_{v=2 s+1}^{\infty} \theta_{v-s-1}
\end{aligned}
$$




$$
\begin{aligned}
\leq & C\left(h_{n}^{\theta}+h_{n}^{-3+\theta} s^{-(a-1)}+h_{n}^{-2} m^{-(a-2)}+h_{n}^{-3} s m^{-b+2}+h_{n}^{-3} s^{-b+3}\right) \\
\leq & C\left(h_{n}^{\theta}+\left(n h_{n}^{3 / 2-\theta}\right)^{-1}+h_{n}^{-2+(1-\theta)(a-2)}\right. \\
& \left.+\left((\log n)^{\delta(a-3) / 4} n h_{n}^{[(1-\theta)(b-2)-3](a-1)-3 / 2}\right)^{1 /(a-1)}\right) .
\end{aligned}
$$

Then, by (A5) and (A6),

$$
\frac{1}{h_{n}} \sum_{v=2}^{\infty}\left|\operatorname{Cov}\left(K\left(\frac{x-\tilde{X}_{1}}{h_{n}}\right), K\left(\frac{x-\tilde{X}_{v}}{h_{n}}\right)\right)\right| \rightarrow 0 .
$$

This completes the proof of (2).

Lemma 4. Assume that assumptions (A1)-(A6) hold. Then, for all Lebesgue points $x$ of $f$,

$$
n h_{n} \operatorname{Varg}_{n}(x) \rightarrow \sigma^{2}(x)
$$

Proof. We will show that

$$
E\left(T_{n}\right)^{2} \rightarrow \sigma^{2}(x)
$$

and

$$
E\left(T_{n}^{\prime}\right)^{2}+E\left(T_{n}^{\prime \prime}\right)^{2} \rightarrow 0
$$

First, consider (7). By using the strict stationarity of the sequence $\left\{Z_{t}\right\}_{t \in \mathbb{Z}}$, we have

$$
E\left(T_{n}\right)^{2}=k \operatorname{Var}\left(\eta_{1}\right)+2 \sum_{1 \leq i<j \leq k} \operatorname{Cov}\left(\eta_{i}, \eta_{j}\right)
$$

Let us establish that

$$
k \operatorname{Var}\left(\eta_{1}\right) \rightarrow \sigma^{2}(x),
$$

and

$$
\sum_{1 \leq i<j \leq k} \operatorname{Cov}\left(\eta_{i}, \eta_{j}\right) \rightarrow 0
$$


By the strict stationarity of the sequence $\left\{Z_{t}\right\}_{t \in \mathbb{Z}}$, we get

$$
k \operatorname{Var}\left(\eta_{1}\right)=\frac{p k}{n h_{n}} \operatorname{Var} K\left(\frac{x-\tilde{X}_{1}}{h_{n}}\right)+\frac{2 k}{n h_{n}} \sum_{1 \leq i<j \leq p} \operatorname{Cov}\left(K\left(\frac{x-\tilde{X}_{i}}{h_{n}}\right), K\left(\frac{x-\tilde{X}_{j}}{h_{n}}\right)\right) .
$$

By Theorem 3 in Chapter 2 of Devroye and Györfi [3], for all Lebesgue points $x$ of $f$,

$$
\frac{1}{h_{n}} \operatorname{Var} K\left(\frac{x-X_{1}}{h_{n}}\right) \rightarrow f(x) \int_{\mathbb{R}} K^{2}(u) d u .
$$

By stationarity and (2), we obtain

$$
\begin{aligned}
\frac{k}{n h_{n}} \mid \sum_{1 \leq i<j \leq p} \operatorname{Cov} & \left(K\left(\frac{x-\tilde{X}_{i}}{h_{n}}\right), K\left(\frac{x-\tilde{X}_{j}}{h_{n}}\right)\right) \mid \\
\leq & \frac{k p}{n h_{n}} \sum_{v=2}^{\infty}\left|\operatorname{Cov}\left(K\left(\frac{x-\tilde{X}_{1}}{h_{n}}\right), K\left(\frac{x-\tilde{X}_{v}}{h_{n}}\right)\right)\right| \rightarrow 0 .
\end{aligned}
$$

On the other hand, by (A2) and (A5),

$$
\begin{aligned}
\frac{1}{h_{n}}\left|\operatorname{Var} K\left(\frac{x-X_{1}}{h_{n}}\right)-\operatorname{Var} K\left(\frac{x-\tilde{X}_{1}}{h_{n}}\right)\right| & \leq \frac{4\|K\|_{\infty}}{h_{n}} E\left|K\left(\frac{x-X_{1}}{h_{n}}\right)-K\left(\frac{x-\tilde{X}_{1}}{h_{n}}\right)\right| \\
& \leq \frac{C}{h_{n}^{2}} E\left|X_{1}-\tilde{X}_{1}\right| \\
& \leq \frac{C}{h_{n}^{2}}\left[E\left(\sum_{r=s+1}^{\infty} a_{r} Z_{1-r}\right)^{2}\right]^{1 / 2} \\
& \leq \frac{C}{h_{n}^{2}} \sum_{r=s+1}^{\infty}\left|a_{r}\right| \rightarrow 0 .
\end{aligned}
$$

The proof of (9) is completed by (11), (12), and (13). 
Now, we will establish (10). Using stationarity and (6), we get

$$
\begin{aligned}
\sum_{1 \leq i<j \leq k}\left|\operatorname{Cov}\left(\eta_{i}, \eta_{j}\right)\right| & \leq k \sum_{l=1}^{k-1}\left|\operatorname{Cov}\left(\eta_{1}, \eta_{l+1}\right)\right| \\
& \leq \frac{p k}{n h_{n}} \sum_{l=1}^{k-1} \sum_{r=l(p+q)-p+1}^{l(p+q)+p-1}\left|\operatorname{Cov}\left(K\left(\frac{x-\tilde{X}_{1}}{h_{n}}\right), K\left(\frac{x-\tilde{X}_{r}}{h_{n}}\right)\right)\right| \\
& \leq \frac{p k}{n h_{n}} \sum_{r=q+1}^{\infty}\left|\operatorname{Cov}\left(K\left(\frac{x-\tilde{X}_{1}}{h_{n}}\right), K\left(\frac{x-\tilde{X}_{r}}{h_{n}}\right)\right)\right| \\
& \leq C \frac{p k s^{2}}{n h_{n}^{3}} \sum_{r=q+1}^{\infty} \theta_{r-s} .
\end{aligned}
$$

Then, by (A4) and (A6), and by choosing $q=2 s$, we obtain

$$
\sum_{1 \leq i<j \leq k}\left|\operatorname{Cov}\left(\eta_{i}, \eta_{j}\right)\right| \rightarrow 0
$$

Now we turn to (8). Observe that

$$
E\left(T_{n}^{\prime}\right)^{2} \leq k \operatorname{Var}\left(\xi_{1}\right)+2 \sum_{1 \leq i<j \leq k}\left|\operatorname{Cov}\left(\xi_{i}, \xi_{j}\right)\right| .
$$

Once again, by stationarity,

$$
k \operatorname{Var}\left(\xi_{1}\right)=\frac{q k}{n h_{n}} \operatorname{Var} K\left(\frac{x-\tilde{X}_{1}}{h_{n}}\right)+\frac{2 k}{n h_{n}} \sum_{1 \leq i<j \leq q} \operatorname{Cov}\left(K\left(\frac{x-\tilde{X}_{i}}{h_{n}}\right), K\left(\frac{x-\tilde{X}_{j}}{h_{n}}\right)\right) .
$$

By (2), (11), and (13), it follows that

$$
k \operatorname{Var}\left(\xi_{1}\right) \rightarrow 0 \text {. }
$$


Then, by the same arguments in (14) and (15), we obtain, for $n$ large enough,

$$
\begin{aligned}
\sum_{1 \leq i<j \leq k}\left|\operatorname{Cov}\left(\xi_{i}, \xi_{j}\right)\right| & \leq C \frac{q k s^{2}}{n h_{n}^{3}} \sum_{r=p}^{\infty} \theta_{r-s} \\
& \leq C \frac{q k s^{2}}{n h_{n}^{3}} \sum_{r=q}^{\infty} \theta_{r-s} \rightarrow 0 .
\end{aligned}
$$

Therefore

$$
E\left(T_{n}^{\prime}\right)^{2} \rightarrow 0
$$

Now, by stationarity,

$$
E\left(T_{n}^{\prime \prime}\right)^{2} \leq \frac{p+q}{n h_{n}} \operatorname{Var} K\left(\frac{x-\tilde{X}_{1}}{h_{n}}\right)+\frac{2}{n h_{n}} \sum_{1 \leq i<j \leq p+q}\left|\operatorname{Cov}\left(K\left(\frac{x-\tilde{X}_{i}}{h_{n}}\right), K\left(\frac{x-\tilde{X}_{j}}{h_{n}}\right)\right)\right|
$$

By (2), (11), and (13), we get $E\left(T_{n}^{\prime \prime}\right)^{2} \rightarrow 0$, which completes the proof of (8).

Proof of Theorem 1. To finish the proof of the theorem, we will establish the asymptotic normality of $g_{n}(x)$. By (8), it suffices to show that

$$
T_{n} \stackrel{d}{\rightarrow} N\left(0, \sigma^{2}(x)\right)
$$

The proof of convergence in (16) consists in using Theorem 7.2 of Billingsley [1], (9) and showing the following two results:

$$
\left|E\left(e^{i t \sum_{j=1}^{k} \eta_{j}}\right)-\prod_{j=1}^{k} E\left(e^{i t \eta_{j}}\right)\right| \rightarrow 0, \quad \forall t \in \mathbb{R},
$$

and

$$
k E\left(\eta_{1}^{2} 1_{\left\{\eta_{1}>\varepsilon \sqrt{k \operatorname{Var}\left(\eta_{1}\right)}\right\}}\right) \rightarrow 0, \quad \forall \varepsilon>0 .
$$


Let us begin by establishing (18). Since $\left|\eta_{1}\right| \leq C p / \sqrt{n h_{n}}$, it follows that

$$
\begin{aligned}
k E\left(\eta_{1}^{2} 1_{\left\{\eta_{1}>\varepsilon \sqrt{k \operatorname{Var}\left(\eta_{1}\right)}\right\}}\right) & \leq C \frac{k p^{2}}{n h_{n}} P\left(\eta_{1}>\varepsilon \sqrt{k \operatorname{Var}\left(\eta_{1}\right)}\right) \\
& \leq \frac{C}{\varepsilon^{2}} \frac{p^{2}}{n h_{n}} .
\end{aligned}
$$

Choose $p=\left[(\log n)^{-\delta(a-3) / 4(a-1)}\left(n h_{n}\right)^{1 / 2}\right]$. Then, we get

$$
k E\left(\eta_{1}^{2} 1_{\left\{\eta_{1}>\varepsilon \sqrt{k \operatorname{Var}\left(\eta_{1}\right)}\right\}}\right) \rightarrow 0 .
$$

Now, let us establish (17).

$$
\begin{aligned}
\left|E\left(e^{i t \sum_{j=1}^{k} \eta_{j}}\right)-\prod_{j=1}^{k} E\left(e^{i t \eta_{j}}\right)\right| \leq & \left|E\left(e^{i t \sum_{j=1}^{k} \eta_{j}}\right)-E\left(e^{i t \sum_{j=1}^{k-1} \eta_{j}}\right) E\left(e^{i t \eta_{k}}\right)\right| \\
& +\left|E\left(e^{i t \sum_{j=1}^{k-1} \eta_{j}}\right)-\prod_{j=1}^{k-1} E\left(e^{i t \eta_{j}}\right)\right| \\
= & \operatorname{Cov}\left(e^{i t \sum_{j=1}^{k-1} \eta_{j}}, e^{i t \eta_{k}}\right)|+| E\left(e^{i t \sum_{j=1}^{k-1} \eta_{j}}\right)-\prod_{j=1}^{k-1} E\left(e^{i t \eta_{j}}\right) \mid .
\end{aligned}
$$

By a repetition of this argument, inequality (19) becomes

$$
\begin{gathered}
\left|E\left(e^{i t \sum_{j=1}^{k} \eta_{j}}\right)-\prod_{j=1}^{k} E\left(e^{i t \eta_{j}}\right)\right| \leq\left|\operatorname{Cov}\left(e^{i t \sum_{j=1}^{k-1} \eta_{j}}, e^{i t \eta_{k}}\right)\right|+\left|\operatorname{Cov}\left(e^{i t \sum_{j=1}^{k-2} \eta_{j}}, e^{i t \eta_{k-1}}\right)\right| \\
+\cdots+\left|\operatorname{Cov}\left(e^{i t \eta_{2}}, e^{i t \eta_{1}}\right)\right| .
\end{gathered}
$$

Thus, by the same arguments as in (14) and (15), we obtain

$$
\left|E\left(e^{i t \sum_{j=1}^{k} \eta_{j}}\right)-\prod_{j=1}^{k} E\left(e^{i t \eta_{j}}\right)\right| \leq C \frac{p k s^{2} t^{2}}{n h_{n}^{3}} \sum_{r \geq q+1} \theta_{r-s} \rightarrow 0 .
$$




\section{Simulations}

Let $\left(U_{t}\right)$ be the $A R(1)$ process defined by

$$
U_{t}=a U_{t-1}+\varepsilon_{t}, \quad t \geq 1,
$$

where $\left(\varepsilon_{t}\right)$ is an identically independent sequence of centered random variables with distribution $\mathcal{N}(0,1)$ and $|a|<1 .\left(U_{t}\right)$ is a Gaussian process, so it is $\not$-weakly dependent. Now let

$$
X_{t}=b X_{t-1}+U_{t-1}, \quad t \geq 0
$$

where $|b|<1$. The variables $X_{t}$ may be expressed in the following form: $X_{t}=\sum_{j=1}^{\infty} b^{j} U_{t-j}$. To illustrate our result, we simulate the $A R(1)$ process $\left(X_{t}\right)$ and compute the kernel density estimator $f_{n}(x)$ for some value $x$. Then we compare the density, $F_{n}(x)$, of

$$
Z_{n}(x):=\sqrt{n h_{n}}\left(f_{n}(x)-E f_{n}(x)\right) / \sigma_{n}(x), \quad \sigma_{n}^{2}(x)=E f_{n}(x) \int_{\mathbb{R}} K^{2}(u) d u
$$

with the density of $\mathcal{N}(0,1)$.

We consider $a=0.8, b=0.5$ and $x=1$, and two values of $n, 100$ and 500. We choose $h_{n}=2.5 n^{-1 / 5}$ which seems optimal and verifies assumptions (A5) and (A6) (since the sequences $\left(a_{r}\right)$ and $\left(\theta_{k}\right)$ decrease geometrically to 0$)$. The graphs below show that the distribution of $Z_{n}(x)$ is Gaussian when $n$ is big enough. 

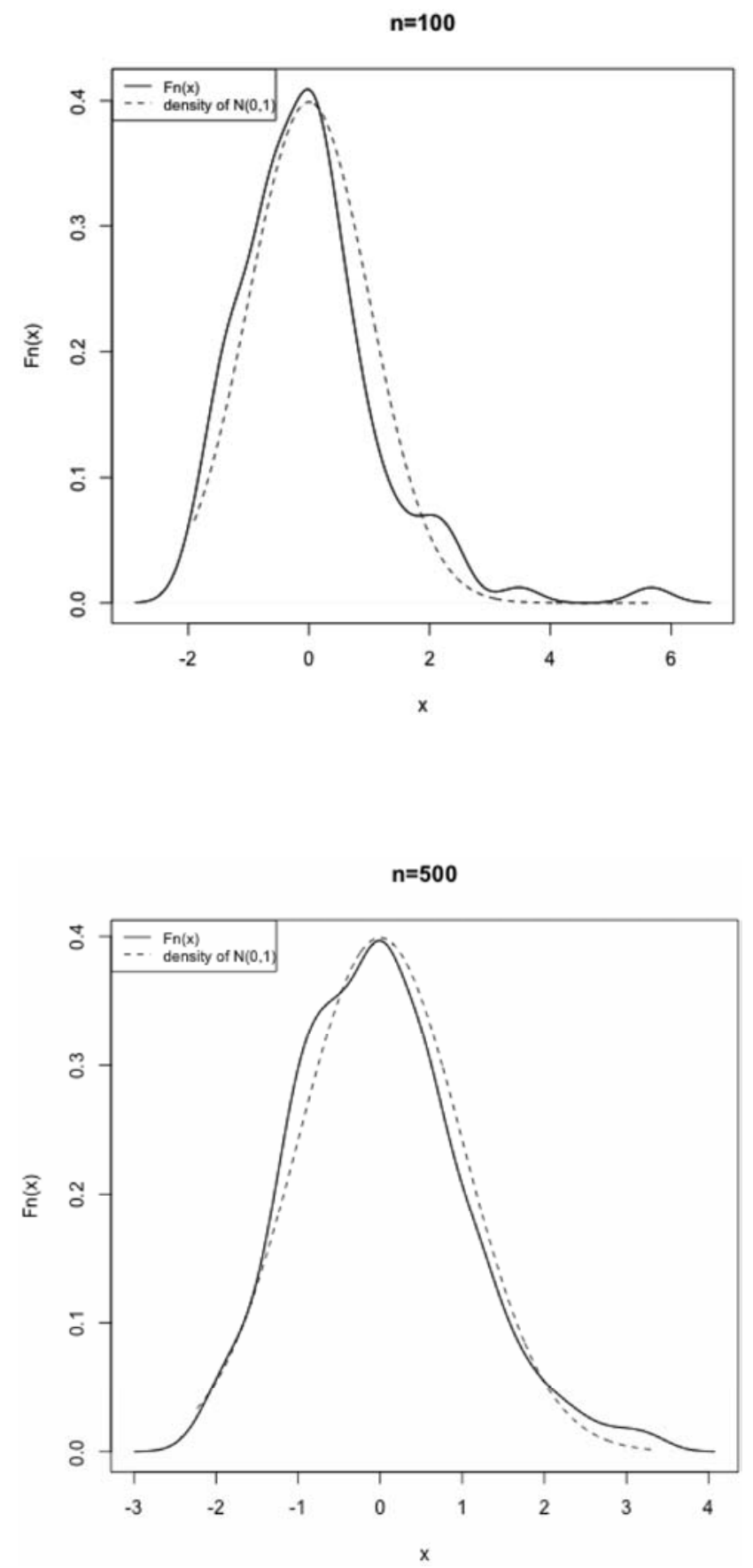


\section{Conclusion}

In this paper, we established the asymptotic normality of the kernel density estimate for linear process with $\psi$-weakly dependent processes. The linear processes with weakly dependent innovations are of great importance in econometrics. We cite for example The ARMA process with ARCH errors discussed in Weiss [13] and Engle [5].

Our method of proof is based on Bernstein's block technique. For this reason we have supposed some conditions on the bandwidth $h_{n}$. For further work, we will check whether other methods allow to suppose weaker conditions on the bandwidth.

\section{References}

[1] P. Billingsley, Convergence of Probability Measures, Wiley, New York, 1968.

[2] K. C. Chanda, Density estimation for linear processes, Annals of the Institute of Statistical Mathematics 35(3) (1983), 439-446.

DOI: https://doi.org/10.1007/BF02481000

[3] L. Devroye and L. Györfi, Nonparametric Density Estimation: The $L_{1}$ View, Wiley, New York, 1985.

[4] P. Doukhan and S. Louhichi, A new weak dependence condition and applications to moment inequalities, Stochastic Processes and their Applications 84(2) (1999), 313-342.

DOI: https://doi.org/10.1016/S0304-4149(99)00055-1

[5] R. F. Engle, Autoregressive conditional heteroscedasticity with estimates of the variance of United Kingdom inflation, Econometrica 50(4) (1982), 987-1007.

DOI: https://doi.org/10.2307/1912773

[6] M. Hallin and L. T. Tran, Kernel density estimation for linear processes: Asymptotic normality and optimal bandwidth derivation, Annals of the Institute of Statistical Mathematics 48(3) (1996), 429-449.

DOI: https://doi.org/10.1007/BF00050847

[7] L. Hamdad, O. Benrabah and S. Dabo-Niang, Kernel density and regression estimations for linear processes with mixing innovations, Journal of Statistics: Advances in Theory and Applications 4(1) (2010), 41-72. 
[8] H.-C. Ho and T. Hsing, On the asymptotic expansion of the empirical process of longmemory moving averages, The Annals of Statistics 24(3) (1996), 992-1024.

DOI: https://doi.org/10.1214/aos/1032526953

[9] T. Honda, Nonparametric density estimation for a long-range dependent linear process, Annals of the Institute of Statistical Mathematics 52(4) (2000), 599-611.

DOI: https://doi.org/10.1023/A:1017504723799

[10] T. Honda, Nonparametric density estimation for linear processes with infinite variance, Annals of the Institute of Statistical Mathematics 61(2) (2009), 413-439.

DOI: https://doi.org/10.1007/s10463-007-0149-x

[11] Z. Lu, Asymptotic normality of kernel density estimators under dependence, Annals of the Institute of Statistical Mathematics 53(3) (2001), 447-468.

DOI: https://doi.org/10.1023/A:1014652626073

[12] A. Schick and W. Wefelmeyer, Pointwise convergence rates and central limit theorems for kernel density estimators in linear processes, Statistics \& Probability Letters 76(16) (2006), 1756-1760.

DOI: https://doi.org/10.1016/j.spl.2006.04.021

[13] A. A. Weiss, Arma models with arch errors, Journal of Time Series Analysis 5(2) (1984), 129-143.

DOI: https://doi.org/10.1111/j.1467-9892.1984.tb00382.x

[14] J. Mielniczuk and W. B. Wu, Kernel density estimation for linear processes, Annals of Statistics 30(5) (2002), 1441-1459.

DOI: https://doi.org/10.1214/aos/1035844982

[15] W. B. Wu, Y. Huang and Y. Huang, Kernel estimation for time series: An asymptotic theory, Stochastic Processes and their Applications 120(12) (2010), 2412-2431.

DOI: https://doi.org/10.1016/j.spa.2010.08.001 